\title{
An Overview of Human Performance Models in Human-Computer Interactions ${ }^{*}$
}

\author{
$\mathrm{Li} \mathrm{Pi}$ \\ Kunming University of Science and Technology \\ School of Information Engineering and Automation \\ Communication Engineering \\ Kunming, China \\ 632166061@qq.com
}

\author{
Jibin Yin \\ Kunming University of Science and Technology \\ School of Information Engineering and Automation \\ Communication Engineering \\ Kunming, China \\ yjb@cnlab.net
}

\begin{abstract}
Human computer interactions (HCI) are the technologies of realizing the dialogue between human and computer in an effective way through computer input and output devices. Human performance model has an important theoretical guidance for the development of human computer interaction. Through the establishment and research on human performance models, we can get the relationship between the movement time (MT) and the index of difficulty (ID), and using the model to evaluate different modes of interactions. This paper will present and sum up the human performance models from diverse interaction modes, and present some research problems of the current research of HCI models.
\end{abstract}

Keywords-Human Computer Interaction (HCI), human performance, modeling

\section{INTRODUCTION}

In traditional interactive interfaces, the validity of Fitts' law has been recognized by the majority of human computer interaction researchers. As a theoretical basis for guiding the design of user interfaces, Fitts' law was widely used to evaluate new technologies, new equipments and new HCI methods as a tool of the theory. It was nearly impossible to compare device performance results from different studies until the Fitts' law model was applied.[1] Fitts' law is also very extensive, and development and evolution of a series of human performance models suitable for different interactive environment, or applied to different input devices, such as

\footnotetext{
*Supported by National Natural Science Foundation of China (61262042)
}

steering law, the model for scrolling, the peephole pointing model and the two-dimensional pointing model. We will illustrate human performance models based on the perspective of the dimension of human computer interaction models. In the end we will present some important problems related to the research of human computer interaction models.

\section{ONE DIMENSIONAL HCI MODELS}

One dimensional HCI models include Fitts' law, the steering law, the peephole pointing model and so on. One dimensional HCI models mainly discuss pointing tasks or trajectory-based tasks which targets were only limited by their width (W). The MT and ID can be affected by the amplitude (A) between the targets and their width (W).

\section{A. Fitts'law}

Fitts' law is the HCI model based on one dimension pointing task. Fitts sought to establish the information capacity of the human motor system. He claimed that electronic signals are analogous to movement distances or amplitudes (A) and that noise is analogous to the tolerance or width (W) of the region within which a move terminates. Loosely based on Shannon's logarithmic expression.[2] The MT for the motor task is:

$$
\mathrm{MT}=\mathrm{a}+\mathrm{b} \log _{2}(2 \mathrm{~A} / \mathrm{W})
$$

From the MT model, we can get the 2A / W mainly impact the ID, the MT is large when the A is large or $\mathrm{W}$ is small. And the MT is linear with ID. So the ID is: 


$$
\mathrm{ID}=\log _{2}(2 \mathrm{~A} / \mathrm{W})
$$

In the experiments of Fitts' law, participates were asked to move a stylus back and forth between two plates as quickly as possible and tapped the plates at their centers in the tapping experiments. In these experiments, there are a total of 16 combinations of A and W. Thus, the ID of each task to tap the target is different. This arrangement of the experiment is commonly called the "Fitts' paradigm."

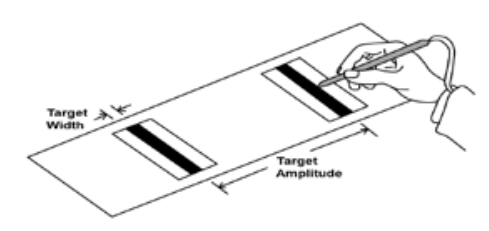

Fig. 1. the tapping experiments of Fitts' law [3]

From the experiments, the average number of errors made in this task was small, only $1.2 \%$ with the lighter stylus and $1.3 \%$ with the heavier one. For each category of W, movement time increased progressively as movement amplitude increased. Likewise for each $\mathrm{A}$, movement time increased progressively as tolerance was decreased. The relations among the various conditions for the lighter and heavier styluses were similar.[3]

\section{B. A Shannon Variation on Fitts' Law}

Recently MacKenzie[4] found that the signal-to-noise ratio in Shannon's theorem corresponds to the ratio of target amplitude to target width in Fitts' analogy. Analogy with Shannon's information theorem, the ratio of the A:W is 1 . The HCI model of Shannon variation of Fitts' law is.

$$
\mathrm{MT}=\mathrm{a}+\mathrm{b} \log _{2}(\mathrm{~A} / \mathrm{W}+1)
$$

In the research of the variations on the Fitts' law. MacKenzie made a comparison between original Fitts' law and variations of the Fitts' law in the table 1.

TABLE I. CORRELATIONS AND INTERCEPTS (MS) FOR FITTS' TAPPING EXPERIMENTS USING EFFECTIVE TARGET WIDTH

\begin{tabular}{|c|c|c|c|c|}
\hline \multirow{2}{*}{ Model } & \multicolumn{2}{|c|}{ Tapping(1 oz) } & \multicolumn{2}{c|}{ Tapping(1 lb) } \\
\cline { 2 - 5 } & $\boldsymbol{r}^{2}$ & Intercept & $\boldsymbol{r}^{2}$ & Intercept \\
\hline Fitts & 0.9907 & -73.2 & 0.9885 & -118.0 \\
\hline Shannon & 0.9938 & -31.4 & 0.9927 & -69.8 \\
\hline
\end{tabular}

As it is shown in table 1 , we can get the $r^{2}$ for the Shannon variation of Fitts' law is larger than the original Fitts' law in the tapping experiments. Thus, the Shannon variation of Fitts' law model addresses several theoretical issues and offers slightly better prediction power than original Fitts' law model.

\section{Steering law}

In trajectory tasks, users often want to move a cursor through the tunnel in a certain amplitude and width. However, it is obvious that Fitts' law addresses only one type of movement.[5] Fitts' law is not an adequate model for trajectory tasks. Accot and Zhai found Steering law on the basis of the Fitts' law, through a rigorous mathematical derivation. The paradigm of the Steering law is:

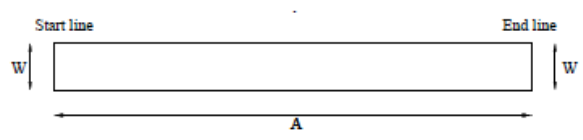

Fig. 2. the paradigm of the Steering law [5]

Through the paradigm, A is the amplitude of the tunnel and $\mathrm{W}$ is for the width of the tunnel. And the model of Steering law is:

$$
\mathrm{MT}=\mathrm{a}+\mathrm{b}(\mathrm{A} / \mathrm{W})
$$

From the nature, the trajectory tasks were composed of numerous small distance pointing tasks. Steering law is the integral derivation from the Fitts' law. Steering law has been proved to be robust. And it has been widely used in many different conditions of trajectory tasks.

\section{Peephole pointing}

Peephole interaction occurs when the contents of a virtual space can not be displayed in a display. In the Peephole pointing, the user can move a display as a window to display the different part in the virtual space. Due to the target is not in the current window, user will move the window to get the target in the scope of the window and select the target. The movement time contains the time moving the window(MD) and the time moving the cursor(MC) .

$$
M T=T_{M D}+T_{M C}
$$

Peephole pointing without prior knowledge, the user does not know the target location. The $\mathrm{T}_{\mathrm{MD} 1}$ was affected by the 
amplitude(A) of the target and the size(S) of the window. $\mathrm{T}_{\mathrm{MD} 1}$ fallows a Fitts' law relationship with A and S. Considering the user will often overshoot the window before MC starts. The effect mean value of target distance is $\mathrm{k}_{1} \mathrm{~S}$ in $\mathrm{T}_{\mathrm{MC} 1}$ and also was affected by the target width(W). The value $k_{1}$ is a constant coefficient.

$$
\begin{aligned}
& \mathrm{T}_{\mathrm{MD} 1}=\mathrm{a}_{\mathrm{D} 1}+\mathrm{b}_{\mathrm{D} 1} \log _{2}(\mathrm{~A} / \mathrm{S}+\mathrm{c}) \\
& \mathrm{T}_{\mathrm{MC} 1}=\mathrm{a}_{\mathrm{C} 1}+\mathrm{b}_{\mathrm{C} 1} \log _{2}\left(\mathrm{k}_{1} \mathrm{~S} / \mathrm{W}+\mathrm{c}\right)
\end{aligned}
$$

Peephole pointing with prior knowledge, MD was a planned movement towards the target to cover it with the window. This is essentially the behavior of an area cursor, also modeled by Fitts' law as reported by Kabbash and Buxton.[6] $\mathrm{MC}$ is a typical task of one dimension pointing with $\mathrm{W}$ is the width of the target and A' is the distance between the cursor and current target. Some researchers showed that in such an action (in the $1 \mathrm{D}$ case) the position of the endpoints $\mathrm{X}$ can be approximated by a normal distribution $\mathrm{N}\left(\mathrm{X}_{0}, \sigma\right)$, where the mean value $\mathrm{X}_{0}$ is the center of the target, and the standard deviation $\sigma=\mathrm{fA}, \mathrm{f}$ being an empirically determined constant.[7,8,9] Therefore A' was approximated by a normal distribution $\mathrm{N}\left(\mathrm{X}_{0}, \sigma\right)$. The mean value of $\mathrm{A}^{\prime}$ is $\sigma \sqrt{2 / \pi}=$ $\mathrm{fA} \sqrt{2 / \pi}=\mathrm{k}_{2} \mathrm{~A}$. The $\mathrm{k}_{2}$ is an empirically determined constant.

$$
\begin{aligned}
& \mathrm{T}_{\mathrm{MD} 2}=\mathrm{a}_{\mathrm{D} 2}+\mathrm{b}_{\mathrm{D} 2} \log _{2}(\mathrm{~A} / \mathrm{S}+\mathrm{c}) \\
& \mathrm{T}_{\mathrm{MC} 2}=\mathrm{a}_{\mathrm{C} 2}+\mathrm{b}_{\mathrm{C} 2} \log _{2}\left(\mathrm{k}_{2} \mathrm{~A} / \mathrm{W}+\mathrm{c}\right)
\end{aligned}
$$

Summarizing the two cases of peephole pointing, we can get the model for peephole pointing.

$$
\left.\mathrm{MT}=\mathrm{a}+\mathrm{b}\left[\mathrm{n} \log _{2}(\mathrm{~A} / \mathrm{S}+1)\right)+(1-\mathrm{n}) \log _{2}(\mathrm{~A} / \mathrm{W}+1)\right](10)
$$

The equation 10 has the best fit with experiment data in all 4 categories, with r2 always greater than 0.9.[10] Thus, the model for peephole pointing is best represented as equation 10.

\section{TWO DIMENSIONAL HCI MODELS}

Two dimensional HCI models mainly discuss the pointing task in the 2D space. In the two dimensional HCI models, the height and the angle of targets can also affect the MT.

\section{A. Extending of Fitts' law}

Fitts' law is inherently a 1D model. In experimental studies, it is typical to set the width of the pointing targets to a set of controlled values, but leave the target height $(\mathrm{H})$ practically at infinity. The extending of Fitts' law will take the target height into consideration. In 2D pointing task, the width is not the only factor of the target to affect the movement time. Therefore, we can use $\mathrm{W}$ and $\mathrm{H}$ to present the different

\begin{tabular}{|c|c|c|c|c|c|c|c|}
\hline \multicolumn{3}{|c|}{ Model } & \multicolumn{5}{|c|}{ Target Width } \\
\hline \multicolumn{3}{|c|}{ STATUS QUO } & \multicolumn{5}{|c|}{ horizontal extent(W) } \\
\hline \multicolumn{3}{|c|}{$\mathrm{W}+\mathrm{H}$} & \multicolumn{5}{|c|}{ sum of width and height } \\
\hline \multicolumn{3}{|c|}{$\mathrm{W} \times \mathrm{H}$} & \multicolumn{5}{|c|}{ area } \\
\hline \multicolumn{3}{|c|}{ SMALLER-OF } & \multicolumn{5}{|c|}{ smaller of width or height } \\
\hline \multicolumn{3}{|c|}{$\mathrm{W}^{\prime}$} & \multicolumn{5}{|c|}{ width along line of approach } \\
\hline \multirow[b]{2}{*}{$\begin{array}{l}\text { Model for } \\
\text { Target Width }\end{array}$} & \multicolumn{2}{|c|}{ ID Range (bits) } & & \multicolumn{4}{|c|}{ Regression Coefficients } \\
\hline & & High & $r^{n}$ & $\underset{(\mathrm{ms})}{S E^{b}}$ & $\begin{array}{l}\text { Intercept, } \\
\text { (ms) }\end{array}$ & $\begin{array}{l}\text { Slope, b } \\
\text { (ms/bit) }\end{array}$ & $\begin{array}{l}\text { IP } \\
(\mathrm{bits} / \mathrm{s})\end{array}$ \\
\hline SMALLER-OF & 1.58 & 5.04 & .9501 & 64 & 230 & 166 & 6.0 \\
\hline$w^{\prime}$ & 1.00 & 5.04 & .9333 & 74 & 337 & 160 & 6.3 \\
\hline $\mathrm{W}+\mathrm{H}$ & 0.74 & 3.54 & .8755 & 99 & 402 & 218 & 4.6 \\
\hline WXH & 0.32 & 4.09 & .8446 & 110 & 481 & 173 & 5.8 \\
\hline STATUS QUO & 1.00 & 5.04 & .8097 & 121 & 409 & 135 & 7.4 \\
\hline \multicolumn{8}{|c|}{$\begin{array}{l}a n=78, p<.001 \\
b \text { standard error of estimate }\end{array}$} \\
\hline
\end{tabular}
relationship while affecting the MT and instead of the width in the 1D Fitt's law.

TABLE II. FIVE EXTENDING TARGET WIDTH OF FITT'S LAW IN 2D POINTING TASKS

Fig. 3. Correlations and regression coefficients for five models for target width [11]

From figure 3 we can get that SMALLER-OF model as well as $\mathrm{W}^{\prime}$ model can be better to explain the relationship between the MT, $\mathrm{W}$ and $\mathrm{H}$. The SMALL-OF model and W' model is:

$$
\begin{aligned}
& \mathrm{MT}=\mathrm{a}+\mathrm{b} \log _{2}[\mathrm{~A} / \min (\mathrm{W}, \mathrm{H})+1] \\
& \mathrm{MT}=\mathrm{a}+\mathrm{b} \log _{2}\left(\mathrm{~A} / \mathrm{W}^{\prime}+1\right)
\end{aligned}
$$

From the equation 11, the $\min (\mathrm{W}, \mathrm{H})$ is the minimum of the target width(W) and target height(H). In equation 11 the MT can only be affected by the small value of the $\mathrm{W}$ and $\mathrm{H}$. From the equation 12, W' is the width of the target along an approach vector. In equation 12, the MT can not only be 
impacted by the $\mathrm{H}$ and $\mathrm{W}$, but also can be impacted by the angle of the movement.

\section{B. Bivariate pointing model}

The bivariate pointing is pointing with simultaneous amplitude and directional constraint. Previous research showed that the $\mathrm{W}$ ' or the smaller of the $\mathrm{W}$ and $\mathrm{H}$ can impact the performance in 2D pointing. However, in the bivariate pointing model, $\mathrm{H}$ also exists effect to the task performance when $\mathrm{W}<\mathrm{H}$. The effect of $\mathrm{H}$ was hidden by the smaller value $\mathrm{W}$ in the previous research when $\mathrm{W}<\mathrm{H}$.

$$
\mathrm{MT}=\mathrm{a}+\operatorname{blog}_{2}\left(\sqrt{(D / W)^{2}+\eta(D / H)^{2}}+1\right)
$$

In equation 13, both a, b and $\eta$ are empirically-determined constant. This equation can represent the result of Hoffmann and Sheikh[12] with the $r^{2}$ larger than 0.99. Compared with bivariate pointing model, the SMALL-OF model ignores the effect of larger parameter of the $\mathrm{W}$ and $\mathrm{H}$. In contrast, previous models, such as the SMALL-OF model, could not explain our empirical findings, particularly the fact that $\mathrm{W}$ influences movement time even if it is greater than $\mathrm{H}$. With the SMALL-OF model, W's influence should vanish if $\mathrm{H}<\mathrm{W}$.[13] Thus the bivariate pointing model has greater predictive power than the SMALL-OF model in the 2D pointing task.

\section{THREE DIMENSIONAL HCI MODEL}

While previous work [11, 12, 13, 14] has resulted in a good understanding of bivariate pointing in 2D space, the same cannot be said for pointing to trivariate targets in 3D. Some researchers $[15,16]$ found that the SMALL-OF model in the $2 \mathrm{D}$ pointing task can be easily extended to 3D environment:

$$
\mathrm{MT}_{\min }=\mathrm{a}+\operatorname{blog}_{2}[\mathrm{~A} / \min (\mathrm{W}, \mathrm{H}, \mathrm{D})+1]
$$

We extend this model to accommodate the possible relative effects of different target dimensions, by assigning weights $\alpha$ and $\beta$ to these parameters, resulting in:

$$
\mathrm{MT}_{\mathrm{Wtmin}}=\mathrm{a}+\operatorname{blog}_{2}[\mathrm{~A} / \min (\mathrm{W}, \alpha \mathrm{H}, \beta \mathrm{D})+1]
$$

However, the equation 14 and 15 suffer from the same problem as the SMALL-OF model. Due to this model only consider the effect of the smallest parameter of target width, height and distance, the model usually ignores the effect of the other two dimensions. Thus, we can extend Zhai's[13] bivariate pointing model to $3 \mathrm{D}$ version.

$\mathrm{MT}_{\mathrm{WtEuc}}=\mathrm{a}+\operatorname{blog}_{2}\left(\sqrt{(A / W)^{2}+\alpha(A / H)^{2}+\beta(A / D)^{2}}+1\right)$

In equation 14 and 16, both of the two models have the same problem. They do not take the angle of movement $\Theta$ into consideration. To accommodate movement angles, all components should also be weighted by an additional parameter $\mathrm{f}_{\mathrm{W}, \mathrm{H}, \mathrm{D}}(\Theta)$ which takes on different empirically determined values dependent on movement angl@. [17] The models are:

$\mathrm{MT}_{\mathrm{Wtmin} \Theta}=\mathrm{a}+\operatorname{blog}_{2}\left[\mathrm{~A} / \min \left(\mathrm{f}_{\mathrm{W}}(\Theta) \mathrm{W}, \mathrm{f}_{\mathrm{H}}(\Theta) \mathrm{H}, \mathrm{f}_{\mathrm{D}}(\Theta) \mathrm{D}\right)+1\right]$

$\mathrm{MT}_{\text {WtEuc } \Theta}=\mathrm{a}+\operatorname{blog}_{2}\left(\sqrt{\mathrm{f}_{\mathrm{W}}(\theta)(A / W)^{2}+\mathrm{f}_{\mathrm{H}}(\theta)(A / H)^{2}+\mathrm{f}_{\mathrm{D}}(\theta)(A / D)^{2}}+1\right)$

In the experiment, we used a 3D volumetric display, which generates a10" spherical 3D volumetric image by sweeping a semitransparent 2D image plane around the Y-axis. The task was reciprocal 3D target acquisition, which required participants to point to two targets back and forth in succession.[17]

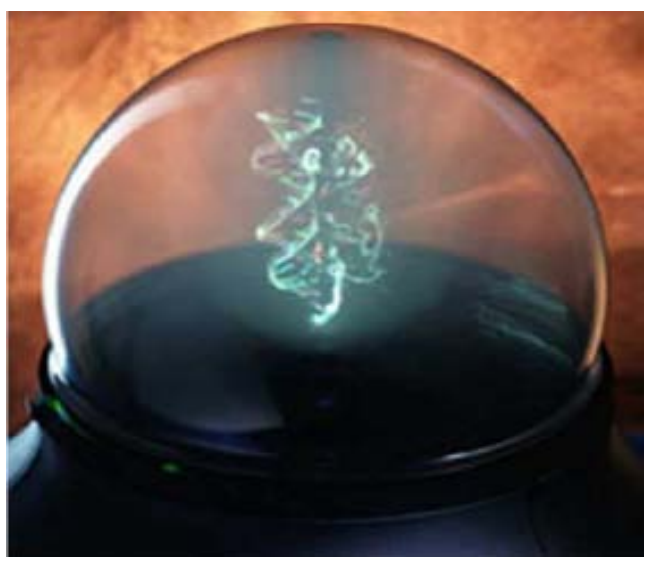

Fig. 4. Volumetric display 

POINTING EXPERIMENTS[17]

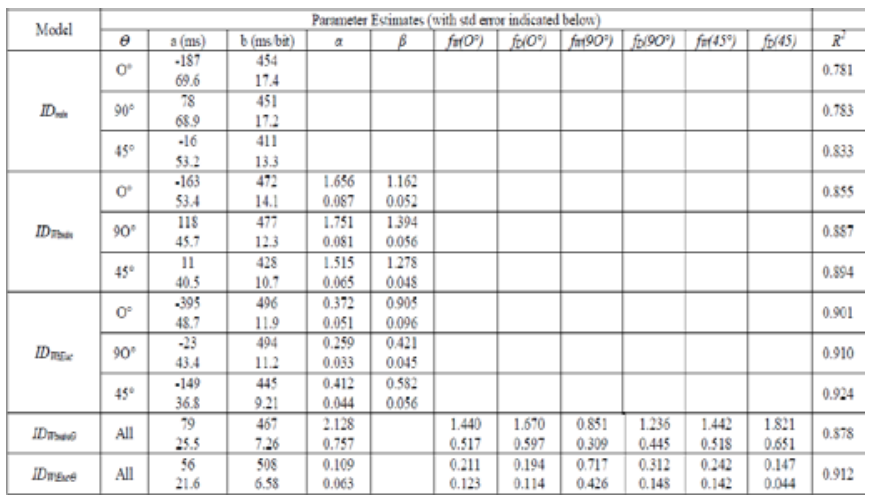

From table 3 we can get the equation 18 is the best fit for the $3 \mathrm{D}$ pointing with $\mathrm{r}^{2}$ is 0.912 . Thus, in the 3D pointing task, the MT is not simply affected by the $\mathrm{W}, \mathrm{H}$ and $\mathrm{D}$. The angle of the movement $(\Theta)$ can impact on the parameter $\mathrm{f}_{\mathrm{W}}(\theta)$, $\mathrm{f}_{\mathrm{H}}(\theta)$ and $\mathrm{f}_{\mathrm{D}}(\theta)$

\section{DISCUSSION AND CONCLUSIONS}

In this paper we presented and summed up the human performance models from the perspective of the dimension. Human performance models are very essential in the field of designing user interface (UI), evaluating of the performance of input or output devices. With the rapid development of the computer technology, numerous methods of human computer interaction have emerged. Such as methods of touch interactions, interactions in the $3 \mathrm{D}$ space and interactions based "pen + touch" input modalties. However, some of these methods still have no appropriate model to evaluate the performance:

First, in the one dimensional pointing tasks, Fitts' law and other variations of Fitts' law only discuss the situation when the start target and the end target appear in the same time. But researchers ignore the asynchronous situation of shown target .

Second, with respect to the peephole pointing model, it only concerns the pointing task in the moving window of the display. But the model ignores the situation when users drag an object through the window and move the object to another place of the display.
Third, with the development of the input devices, we can use the gesture to interact with the device in the 3D space. There is no suitable model to evaluate the performance of the gesture tasks in the 3D space.

Fourth, Fitts' law can evaluate performance of one dimensional pointing tasks in the touch interfaces. However, with the development of the touch interactions, users can use the touch and pen interactions at the same time to achieve some complex operations. What model is suitable for the "pen + touch" interactions is another issue in the research of $\mathrm{HCI}$ models.

With the extending research of Fitts' law, the models of these methods of interactions will be found in future studies.

\section{References}

[1] Card, S.K., English, W.K., \& Burr, B. J. (1978).Evaluation of mouse, rate-controlled isometric joystick,step keys and text keys for text selection on a CRT.Ergonomics, 21(8), 601-613.

[2] MacKenzie, I. S. (1992). Fitts' law as a research and design tool in human-computer interaction. Human-Computer Interaction, 7, 91-139. doi: 10.1207/s15327051hci0701_3

[3] Fitts, P.M. The information capacity of the human motor system in controlling the amplitude of movement. Journal of Experimental Psychology, Vol.47, pp. 381-391, 1954.

[4] MacKenzie, I. S. (1989). A note on the information-theoretic basis for Fitts' law. Journal of Motor Behavior, 21, 323-330.

[5] Accot, J. and Zhai, S. (1997). Beyond Fitts' Law: Models for trajectory-based HCI tasks. ACM CHI Conference. p. 295-302.

[6] Kabbash, P. and Buxton, W. (1995). The "Prince" technique: Fitts' law and selection using area cursors. ACM CHI Conference. p. 273-279.

[7] Grossman, T. and Balakrishnan, R. (2005). A probabilistic approach to modeling two-dimensionalpointing. ACM Transactions on Computer Human Interaction, 12(3). p. 435-459.

[8] Meyer, D.E., Smith, J.E., Kornblum, S., Abrams, R.A., and Wright, C.E. (1988). Optimality in human motorperformance: Ideal control of rapid aimed movements.Psychological Review, 95. p. 340-370.

[9] Welford, A. (1968). The fundamentals of skill. London: Methuen.

[10] Xiang Cao, Jacky Jie Li, Ravin Balakrishnan. (2008). Peephole pointing: Modeling acquisition of dynamically revealed targets. Proceedings of CHI 2008 - the ACM Conference on Human Factors in Computing Systems. p. 1699-1708.

[11] I. S. MacKenzie and W. Buxton. Extending Fitts' law to two-dimensional tasks. In Proceedings of the ACM SIGCHI Conference on Human Factors in Computing Systems, pages 219-226, 1992.

[12] E. R. Hoffmann and I. H. Sheikh. Effect of varying target height in a Fitts' movement task. Ergonomics, 37(6):1071-1088, 1994.

[13] Johnny Accot, Shumin Zhai. Refining Fitts' law models for bivariate pointing. Conference: Computer Human Interaction - CHI , pp. 193-200, 2003

[14] Crossman, E. (1956). The measurement of perceptual load in manual operations. PhD Thesis. U. Birmingham.

[15] Ware, C., \& Balakrishnan, R. (1994). Reaching for objects in VR displays: Lag and frame rate. ACM TOCHI. 1(4). p. 331-356.

[16] Ware, C., \& Lowther, K. (1997). Selection using a oneeyed cursor in a fish-tank VR environment. ACM TOCHI. 4(4). p. 309-322.

[17] Tovi Grossman and Ravin Balakrishnan. 2004. Pointing at trivariate targets in 3D environments. In Proceedings of the SIGCHI conference on Human factors in computing systems. ACM, New York, NY, USA, 447-454. 